\title{
SISTEMÁTICA DE LAS PLANTAS MEDICINALES DE USO FRECUENTE EN EL ÁREA DE IQUITOS
}

Rodolfo Vásquez ( $\left.{ }^{*}\right)$

\section{RESUMEN}

En éste artículo, se reporta la existencia de 105 especies de plantas medicinales, que los habitantes de Iquitos y alrededores usan frecuentemente en el tratamiento de ciertas dolencias; la idea de esta contribución es aclarar ciertos nombres científicos que erróneamente se vienen usando para las plantas medicinales de uso folklórico.

\section{ABST RACT}

This paper reports 105 species of medicinal plants, used frequently in Iquitos area; the idea is to expleain the correct $L$ atin names.

Palabras claves: Plantas medicinales, sistemática, Iquitos, Perú.

\section{INTRODUCCION}

El esclarecimiento de la nomenclatura botánica correcta de las especies corrientemente usadas en la medicina popular en el área de Iquitos, podría constituir un primer paso para empezar a trabajar con las plantas del área, llamándolas por sus verdaderos nombres, no solamente en el campo de la medicina popular, como fuente de datos para la búsqueda de principios activos utilizables a corto plazo, sino también en otras áreas de las ciencias aplicadas; claro está sin olvidar los nombres vernácula res, utilizándolos adecuadamente como auxiliares en la determinación taxonómica. De una correcta nomenclatura botánica de las plantas medicinales, dependen muchos estudios químicos, farmacéuticos, estudios de principios activos eficaces en la medicina, estudios antropológicos, históricos, socio-económicos y agronómicos y, desde otro punto de vista, como una base fundamental para el fortalecimiento de la medicina alternativa.

Investigador de campo asociado - Missouri Botanical Garden, Proyecto Flora del Perú, A partado 280Iquitos Perú 
Por estas razones, La determinación botánica de las plantas medicinales de la amazonía peruana, constituye un punto crítico para las investigaciones farmacológicas, químicas y preclínicas, la que se dificulta aún más por los problemas siguientes:

Escasez de literatura especializada.

Escaso material de comparación en los herbarios.

Falta de taxónomos especialistas.

\section{ANTECEDENTES}

L as plantas medicinales del trópico constituyen a mediano plazo y con un buen estudio de factibilidad, una fuente considerable de ingresos para la región; al hacer un análisis de este aspecto, se observa que, según Farnsworth \& Soejarto (1985), en el período de 1959-1973, el 25.36\% de las prescripciones examinadas, contenían uno 0 mas principios activos derivados de plantas superiores tropicales; el valor de estas prescripciones en el mundo, según estimación de CMR (1987), representó en ese año 28 billones, y en 1990,45 billones de U.S. dólares, Sitting (1988), calculó para ese año, 100 billones y Príncipe (1989), calculó para el año 1985, 150 billones de U.S. dólares (Duke, 1991). Respecto a esto, el mismo autor, afirma que no es el $25 \%$ que generalmente se asigna, sino el $125 \%$ las prescripciones que contiene como mínimo un derivado de plantas superiores tropicales; de este porcentaje, se asume que solo la mitad del valor de las prescripciones corresponde a plantas tropicales superiores, o sea el $6.25 \%$; la otra mitad, corresponde a derivados minerales sintéticos, bacterias, animales o plantas inferiores. Si en el mundo el valor de las medicinales equivale a 100 U.S. billones, se asigna pues que 6.25 U.S. billones de dólares por año corresponden a productos derivados de plantas tropicales; sin embargo el valor de los productos naturales crudos es despreciable, el cual alcanza sólo 6.25 millones de dólares U.S. Duke (1991).Drogas naturales, de amplia demanda, como vincristina, vinblastina, reserpina, quinina, quinidina, pilocarpina, y otras ilegítimas, como la cocaína, provienen del bosque tropical. Esto da una idea de la importancia de nuestra región como fuente productora de medicamentos. Duke (1989), compiló una lista de las 250 especies de plantas medicinales más importantes del mundo, de las cuales la mitad corresponde a plantas tropicales, esta lista incluye a plantas silvestres o cultivadas de la región, las que se mencionan a continuación:

Ananas camosus, Banisteriopsis capi, Brumfelsia sp. Camelia sinensis, Cannabis sativa, Carica papaya, Catharanthus roseus, Cephaelis ipecahuana, Chenopodium ambrosoides, Chondodendron tomentosum, Cinchona Cissampelus pareíra, Citrus spp., Copaifera sp., Cotalaria sessiliflora, Curcuma longa, Datura stramonium, Digitalis spp., Erythroxylum coca, Jatropha curcas, Melissa officinalis, Momordica charantea, Mucuna pruriens, Musa sp., Myroxylon balsamum, Nicotiana tabacum, Papaver somniferum, Passíflora spp., Paullinia cupana, Physalis spp., Pilocarpus spp., Plantago spp., Quassia amara, Rauvolfia spp., Ricinus conmunis, Ruta graveolens, 
Smilax spp., Strychnos spp., Tabebuia spp., Tecoma stands, Theobroma cacao, Zea mays y Zingiber officinales.

\section{METODOLOGIA}

Desde hace algunos años, dentro del programa de actividades del proyecto Flora del Perú, se han venido tomando datos sobre los usos de las plantas de la región; mediante entrevistas a nativos, campesinos, madereros, vendedores y usuarios de plantas medicinales; mediante Revisión de literatura y Consulta de Herbario.

\section{RESULTADOS}

Como resultado de este trabajo, se observó que en el área de Iquitos, se practica la medicina folklórica en dos modalidades principales:

Medicina folklórica regional. Basada en el uso de plantas medicinales silvestres y cultivadas, productos animales, así como talismanes y oraciones. Esta a su vez, puede ser: supersticiosa, realista y mixta.

Medicina folklórica foránea. Basada en el uso de plantas medicinales traídas de la costa y sierra, unas pocas regionales, con productos animales y talismanes.

Desde el punto de vista científico y de utilidad para estudios fotoquímicos, se ha elaborado una lista de las plantas vasculares, de uso frecuente en el área de Iquitos, en la medicina folklórica regional realista y mixta.

Para evitar mayores confusiones, no se incluye la ubicación de la familia en su respectivo orden, ya que esto está bien discutido en Becker (1973), donde se señala los sistemas de clasificación de las angiospermas de acuerdo con Cronquist, Takhtajan, Thorne, Engler, Hutchinson y Bentham \& Hooker.

Seguidamente, se muestran las plantas medicinales de uso frecuente en el área de Iquitos, pero antes se deberá tomar en cuenta que:

1. La primera línea más oscura, corresponde al nombre latino correcto y su respectiva familia. NC.

2. La segunda línea, corresponde a/o los nombres latinos, si los hubiere, que corrientemente se atribuyen a dicha planta. O.N .

3. La tercera línea corresponde al nombre (s) vernacular(es). Ny.

4. L a cuarta línea, corresponde al estado natural. EN .

5. La quinta línea, corresponde al uso, sin especificar partes que usan, ni preparación ni dosificación. $U$.
NC. :
$A b L$ rufescens Auble
M enispermaceae
QN
A buta, grandifolia
M enispermaceae 


$\begin{array}{lll}\text { Ny. } & : & \text { A buta, abuta macho } \\ \text { EN. } & : & \text { Silvestre } \\ \text { U. } & : & \text { A ntiartrítico, antirreumático, para la fertilidad, aditivo. }\end{array}$

\section{Alchornea castaneifolia (Willd) J uss}

Euphorbiaceae

Inpuru.

Silvestre.

A ntiartrítico, antirreumático.

\section{A nacardium occidentale $\mathbf{L}$}

A nacandiaceae

Casho, marañón.

Cultivado

A ntidiarreíco, antiséptico, en cardiopatía.

\section{Arrabideae chica (H BK) Verl.}

Bignoniaceae

Puca panga.

Silvestre y cultivado.

A ntiinflamatorio, contra enfermedades de la piel.

\section{Artocarpus altilis (park) Fosberg.}

Moraceae

A ntocarpus incisa

M oraceae

Pandisho, pan del árbol.

Cultivado.

Para hernias recientes, extracción de gusanos de la piel.

\section{Banisteniopsis caapi (Spr. ex G risb.) M orton}

Banisteriopsis muricata

Baaisteria caapi

A yaliuascam yagé.

Silvestre y cultivado.

Alucinógeno, adivinatorio, purgas, abluciones.

Brosimum acutifollum ssp. abovatum (Ducke) M oraceae

CC Berg

Tamamuni, mururé.

Silvestre.

A ntirreumático, antiartrítico, tónico, afrodisíaco.

$\begin{array}{ll}\text { Brosimum ubescens Taubert. } & \text { M oraceae } \\ \text { Brosiuni paraense } & \text { M oraceae }\end{array}$

Palisangre.

M alpighiaceae

$M$ al piphiaceae

$M$ al pighiaceae

Silvestre.

A ntiartnítico, antirreumático, fortificante, para descensos. 
Brugmansia suaveolens (HBK ex Wiild) Bercht \& Prsl. Solanaceae Datura sp.

Solanaceae

Toé, maricahua.

Cultivado.

Alucinógeno, purgas.

Brunfelsia grandiflora ssp. schultesii Plowman

Solanaceae

B. mire Monach

Colanacear

Brumfelsia bonodora Loganiceae

Sanango durum

A pocinaceae

Tabernaemontana sananho

Sanango, chiricsanago.

Cultivo y silvestre.

A ntidiartrítico, antirreumático, purgas.

C ampsiandra angustifolia Spring ex Benth

Fabaceae

Campsiandra laurifolia

Fabaceae

H uacapurana.

Silvestre.

A ntiartrítico, antirreumático, para resfríos.

\section{Cassia reticulata $\mathbf{W}$ ill $\mathbf{d}$}

Fabaceae

Retama.

Silvestre.

Problemas del hígado, riñones, acidez, antimicótico.

Chenopodium ambrosioides $L$, var.anthelmin ticum A maranthaceae

Paico.

Cultivado.

Vermífugo, para cólicos, febrífugo.

Citrus limon (L) Burm.

Limón.

Cultivado.

Problemas respiratorios, sinusitis, conjuntivitis, febrífugo.

Copaif era reticulata Ducke

Fabaceae

Copaifera officinalis

Fabaceae

Copaifera multijuga

Fabaceae

Copaiba.

Silvestre.

A ntiartrítico, antirrcumático, dolores musculares.

Costus arabicus $\mathbf{L}$.

Costaceae

C. spicatus R osc.

Costaceae

Cañagre.

Silvestre.

Febrígo, bronquitis, problemas urinarios. 
C rescentia cuj cte $L$.

Bignoniaceae

Huingo, tutumo, pate.

Cultivado.

Bronquitis, antidiarreico.

Croton lechleri M uell-Arg.

Euphorbiaceae

Croton draconoides

Euphorbiaceae

Cmton erythrochílus

E uphorbiaceae

Sangre de drago, sangre de grado.

Silvestre.

Cicatrizante, úlceras, lavados vaginales.

\section{C urcuma L onga $\mathbf{L}$}

Zinnziberaceae

Guisador, azafran, palillo.

Cultivado.

Hepatitis.

Cymbopogon citratus (DC) Stapt.

Poaceae

Y erba luisa.

Cultivado.

Cólicos, resfríos.

Cyperus articulatus $L$.

Cyperaceae

Piripiri de víbora.

Cultivado.

A ntiofídico.

Desmodium adscendens (Sw) DC.

F abaceae

A mor seco.

Silvestre.

Tranquilizante, encantamientos, para lavados vaginales.

\section{Dracomtium loretense K rause}

A raceae

Jergón sacha.

Silvestre y cultivado.

Para las úlceras, para las pal pitaciones 'pulsario'.

E leuterme plicata Herb.

Iridaceae

Eleuterine bulbosa

Iridaceae

$Y$ ahuar piripiri.

Cultivado.

A ntidisentérico, antidiarreico.

\section{E rythrina fusca L our.}

Fabaceae

Erythrina glauca

Fabaceae

A masisa.

Silvestre y cultivado.

A ntiinflamatorio, antirreumático, antifebrffugo.

E uterpe precatoria Mart.

A recaceae 
Euterpe oleraceae

A recaceae

Huasaí.

Silvestre.

Para los riñones, cicatrizante en hepatitis.

Ficus insipida Willd ssp. Insipida

M oraceae

Ficus anthelmintica

M oraceae

Pharmacosycea anthelmintica

M oraceae

Ojé, doctor ojé.

Silvestre.

Vermífugo, tónico.

Genipa americana L .

Rubiaceae

Huito, huitol.

Silvestre y cultivado.

Bronquitis, se asegura que es abortiva.

G ossypium barbadense $\mathbf{L}$.

M alvaceae

Gossypium peruvianum

$M$ alvaceae

Algodón, algodonero.

Cultivado.

A ntiséptico, ocitócico.

G ynerium sagitarum (Aubl) Beaux.

Poaceae

Cañabrava.

Silvestre.

A ntiasmático, contra el bocio, diurético.

Hibiscus abelmoschus $L$. $M$.

$M$ alvaceae

Hibiscus moschtus

$M$ alvaceae

A ya murillo, mishó isma.

$M$ alvaceae

Bronquitis, tos.

H imatanthus sucuuba (Spruce) W oods.

A pocynaceae

Bellaco caspi.

Silvestre.

Contra las hernias, dolores lumbares, úlceras gástricas.

$\mathrm{H}$ ymenaea oblongifolia $\mathrm{H}$ uber

Fabaceae

$H$. palustris (Ducke) L ee. et. Lauguh

Fabaceae

A zúcar huayo.

Silvestre

A ntiartrítico, antirreumático, antidiarreico.

Hyptis mutabilis (R ich) Briq.

Labiatae

A lbaca cimarrona.

Silvestre.

Febrífugo, cólicos, abluciones. 
J atropha gossypifolia L .

Euphorbiaceae

Piñón negro, piñón colorado.

Cultivado.

A ntiséptico, para gastritis, acidez.

J essenia batauia (mart) Burret. ssp. batua

A racacea

Jessenia polycarpa

Araceae

Hungurahui, unguahui.

Coadyuvante en el tratamiento de TBC.

K alanchoe pinnata (lam) Pers.

Crasulaceae

Bryphyllum pinnatum

Crasulaceae

Hoja del aire.

Cultivado.

A ntiinflamatorio, uretritis, febrífugo.

\section{L aportea aestuans (L) C hew.}

Urticacea

U rera caracasana

Urticacea

Ishanga colorada, ortiga.

Silvestre.

Diurético, para aliviar los dolores reumáticos.

Lepianthes peltata (L) R af.

Piperaceae

Photormophe peltata (L) M iq.

Piperaceae

Santa maría.

Silvestre

Diurético, febrífugo, vómito, antiinflamatorio.

\section{Lippia alba (M ill) Neb.}

V erbenaceae

Pampa orégano.

Silvestre y cultivado.

Contra las náuseas, vómitos, abluciones, contra el insomnio.

L uffa operculata (L) Cogn.

Cucurbitaceae

Esponjilla.

Silvestre y cultivado.

Para sinusitis, contra los callos, celulitis.

M adura tinctorea (L) G aud.

M oraceae

Chlorophora tinctorea

M oraceae

Insira, insira amarilla.

Silvestre.

Cicatrizante, dolor de dientes.

M alachra capitata $\mathbf{L}$.

M alvaceae

$M$ alva. 
Silvestre y cultivado.

U retritis, gastritis, ardor de estómago.

M ansoa alliacea ( $L$ am) A. G entry

Bignoniaceae

M. humennea (DC ) A. G entry

Bignoniaceae

M. standleyi (Steym) A. G entry

Bignoniaceae

A denocal ymmaa alliaceum

Bignoniaceae

Pseudocalymma alliaceum

Bignoniaceae

Pachyptcra hymenaea

Bignoniaceae

A jo sacha, ajo macho.

Silvestre y, cultivado.

A ntirreumático, antiartrítico, purgas, abluciones.

M aquira coriacea (K arst) C C.B erg.

M oraceae

Clarisia nitida

M oraceae

Clarisia biflora

M oraceae

Capinurí.

Silvestre.

Para tratar las hernias, luxaciones.

M artinella ovovata(HBK Bur. \& K.)

Biguoniaceae

Euphorbia cotinoides

Euphorbiaceae

Y uquilla.

Silvestre

Conjuntivitis, irritación de los ojos.

M aytenus macrocarpa (R\& P). Briq.

Celastraceae

M. Krukovii A.C. Smith.

Olacaceae

Heisteria pallida

Erythroxylacacea

Erythroxylum sp.

Chuchuashi, chuchuhuasi, chuchuasha.

A ntirreumático, contra los resfríos, para después del parto, A ntidiarreíco.

M imosa polydactyla Humb. \& Bompl.ex Willd.

Fabaceae

Mimosa pudica

Fabaceae

M imosa nigra

F abaceae

Sensitiva, amor dormido.

Silvestre.

Contra el insomnio, tranquilizante.

M omor dica charantea $L$.

Cucurbitaceae

Papailla

Silvestre.

Contra los cólicos, febrífugo, vómito, para hepatitis. 
M ucuna rostrata Benth.

Fabaceae

$\checkmark$ aca ñahui, corpus sacha.

Silvestre.

Diurético, venereas "mula".

Nicotiana tabacum L.

Solanaceae

Tabaco.

Cultivado.

Para extraer gusanos de la piel, abluciones, purgas.

O cimum macranthum Willd.

L abiatae

Pichana albaca.

Silvestre y cultivado.

Contra la gripe, febrífugo, en abluciones.

Ormosia coccinea J acq.

Fabaceae

H uayruro colorado.

Silvestre.

Para las hemorroides, purgas.

Passiflora nitida HBK

Passiflora ligularis

Passifloraceae

Granadilla.

Passifloraceae

Silvestre y cultivada.

A ntiséptico, para "purificar la sangre".

Persea americana Mill

L auraceae

Palta, palto.

Cultivada.

A ntidiarrico, abortivo.

Petiveria alliacea $\mathrm{L}$.

Phitolaccaceae

M ucura, micura.

Cultivado.

A ntiespasmódico, febrífugo, purgas, abluciones.

Phthirusa adunca (M eyer) M aguire

L oranthaceae

Phoradendron 2spp.

L oranthaceae

Oryctanthus $2 \mathrm{spp}$.

L oranthaceae

Suelda con suelda, pisho isma

Silvestre.

Tratamiento de fracturas, luxaciones para después del parto. 
Phyllanthus stipulathus (R ef) Webster

P. urinaria $L$

Phyllanthus niruri

Chanca piedra.

Silvestre.

Cálculos renales y del hígado.

Piper adundum $\mathbf{L}$.

Cordoncillo.

Silvestre.

M alestar estomacal, purgas.

Piper aff. Callosum

Guayusa.

Cultivado.

Tranquilizante, contra la apatía.

\section{Plantago mayor $\mathbf{L}$.}

L lantén.

Cultivado

Problemas del hígado, antiséptico.

\section{Pogostemon heyneanus Benth}

Orisia.

Cultivado.

Problemas del corazón, estómago, abluciones.

Polypodium decumanum Willd.

Phlebodium decumanum

Calaguala, huayhuashi shupa, coto shupa.

Silvestre.

Febrífugo, tos ferina, malestares renales.

Priva lappulacea (L) Pers.

Puspo quihua, bolsa quihua.

Silvestre

A ntiçonceptivo.

Psidum guayaba $L$.

Guayaba.

Cultivado.

A ntidiarreico, durante la menstruaclofl.

\section{Psychotria viridis R \& P.}

$Y$ agé, tupamaqui.

Silvestre.

A ditivo en las purgas.

\section{$R$ uta graveolens $L$.}

$R$. C halepensis L .

Ruda.
Berbenaceae

Euphorbiaceae

Euphorbiaceae.

Euphorbiaceae

Piperaceae

Piperaceae

Plantaginaaceae

Labiatae

Polypodiaceae

Polypodiaceae

M yrtaceae

Rubiaceae

Rutaceae

Rutaceae 
Cultivado.

A ntiespasmódico, cefáleas, ocitócico, abluciones.

Sambucus mexicana Presl. var. bipinnata (S\& C) Sch. Capriofoliaceae Sauco.

Cultivado.

Cólicos, uretritis, gripe.

\section{Scoparia culcis L.}

Ñuco pichana.

Silvestre.

Febrífugo, hemorroides, malestares renales, vómito.

Semia aff. bacillaris (M abr.) C.B.

Scrophulariaceae

$M$ ataro, mataro chico.

Silvestre.

A ntimicótico, antiinflamatorio.

Siparuna guianensis Aubl.

M onimiaceae

Isula huayo, picho huayo.

Silvestre.

Antimicótico.

Solanum mamosun L.

$V$ aca chucho, tinctona.

Fabaceae

Silvestre.

A ntiséptico, antimicótico.

Solanum sesilliflorum Dun.

Solanaceae

Cocona.

Cultivado.

A ntiséptico, contra la acarosis.

Spondias mombin L.

Solanaceae

S. venulosa $\mathrm{M}$ art. ex Engl.

A nacardiaceae

U bos, ushum.

A nacardiaceae

Silvestre y cultivado.

A ntidisentérico, para lavados vaginales

Swaxtzia polyphylla A.P.C

Fabaceae

Cumaseba.

Silvestre.

A ntirreumático, para después del parto.

Tabebuia chrysanta (J acq) Nichols.

Bignoniaceae

T. incana A. G entry

T. ochraceae ssp. heter opoda(DC)A.G entry

Bignoniaceae

Bignoniaceae

Cariniana dccandra

L ecythidaceae

Bucida spp.

Combretaceae

Terminalia spp.

Combretaceae

Tahuarí negro, tahuarí amarillo. 
Silvestre.

Para cáncer, diabetes.

Smilax regelii Killip et Morton.

Liliaceae

Smilax utilis

Smilax officinalis

Zarzaparrilla.

Silvestre.

A ntiinflamatorio, antirreumático.

Tagetes erecta $L$.

A steraceae

Rosa sisa.

Cultivado.

Cólicos, abluciones.

Teliostachya lanceolata Nees

A canthaceae

Blechum sp.

A canthaceae

Toé negro.

A ditivo, purgas.

Tynanthus panurensis (B ur) G mell.

Rubiaceae

Garabato, uña de gato.

Silvestre.

A ntiartrítico, antirreumático, contra el cancer, diabetes.

Unonopsis floribunda Diels.

A nnonaceae

Icoja, icoja negra.

Silvestre.

A ntirreumático, antiartrítico, antidiarreica.

Vetiveria zizanioides (L) Nash.

Poaceae

Patichuli, pachulí.

Cultivado.

Fortificante del pelo.

\section{Verbena littoralis}

V erbenaceae

Verbena negra.

Cultivado y silvestre.

Para purificar la sangre.

Vismia angusta $\mathbf{M}$ iq.

H ypericaceae

V. lateriflora Ducke

Hypericaceae

V. macrophylla HBK.

Hypericaceae

Vismia spp.

Cuttiferae

Pichirina.

Silvestre.

A ntimicótico. 
Zingiber officinales Rosc.

Zinziberaceae

Gengibre.

Cultivado.

A ntirreumático, vigorizante masculino, antidiarréico.

Se logró catalogar 103 especies de uso frecuente, en el área de Iquitos, determinadas hasta nivel específico y dos especies sólo conocidas por el nombre vernacular "renaquilla" y "lancetilla", ambas de uso difundido; la primera como antirreumático y la segunda como febrífugo, considerando estas adiciones se tiene un total de 105 especies que por falta de muestra, no se llegó a determinar.

\section{DISCUSION}

Aún siendo pocas las especies de uso frecuente en el área, persisten algunos errores en la nomenclatura botánica; algunos graves, como el caso de Tabebuia spp, donde deliberadamente los extractores colectan cortezas de Lecithydaceas y Combretáceas, para aumentar el volumen de sus ventas. Otros errores son cometidos por las personas que llevan datos, sin tomar muestras de herbario, para su posterior discernimiento, solo en base de nombres vernaculares y consiguen su (correspondiente) nombre científico en los catálogos o literatura que tratan estos temas, tales como Maquinaria coraicea "capinurí", la que frecuentemente aparece en la literatura como Clarisia nitida o clarisia biflora, también M artinella ovovata "yuquilla", se encuentran algunos reportes como Euphorbia cotinoides, o también Brumfelsia grandiflora sp. Schultesii"chiric sanango", se le conoce como Brunfelsia bonodira, una especie que no se encuentra en la zona, o en el peor de los casos, se le encuentra como Sanango durutn, o como Tabernaemontana sananho. Otros errores frecuentes, pero de menor trascendencia, se debe a que se siguen usando muchos nombres que pasaron a ser sinónimos; esto, debido generalmente a la falta de disposición de material bibliográfico, entre estos se pueden tomar como ejemplos a: Mansoa sp. "ajo shacha" conocido frecuentemente como Adenocalymma sp, Pseudocalymma sp., Pachyptera sp, y de igual modo Lepianthes peltata (L) Raf. "santa maría", conocido como Pothormorphe peltata (L) M iq.

Todos estos errores frecuentes, no solo en las plantas medicinales sino también en todos los trabajos que traten de plantas amazónicas, serán solucionados en la medida que cada vez que se realice un estudio, se tomen muestras y sean depositadas en uno 0 más herbarios, las que deberán ser determinadas in situ, o enviadas a los especialistas, dentro o fuera del país. 


\section{BIBLIOGRAFIA}

AYALA, F.F. \& W.H. LEWIX, 1978. Drinking the south A merican Hallucinogenic A yahuasca, ECONOMIC BOTANY 32: 154-156.

1984. N otes on some medicinal and poisonous plant of A mazonian Perú, ADVANCES IN ECONOMIC BOTANY 1:1-8.

BECKER, K.M., 1973. A comparison of Angiosperm classification Systems, TAXON 22 (1): $19-50$.

BERG, M.E. VAN DEN, 1982. A proveitamiento alternativo de essencias florestais A mazonicos, SILVICULTURA EMS. PAULO. 16A, 1:226-231.

1983. Etnobotanica-A experiencia Brasileira, do MuseuGoeldi, 2do. Simposio Nacional de F armacologia e Quimica de productos naturais, ANAIS, 195-201.

-1984. V er-o-Peso: The Tthnobotany of an A mazonian M arket, ADVANCES IN ECONOMIC BOTANY 1:140-149.

1986. Contribuqáo ao conhecimento da flora medicinal do Marahnáo, ler Simposio do Trópico U mido, A NAIS, V ol. II, 119-125.

1986. Plantas medicinais do Amazonías, ler. Simposio do Trópico U mido, ANAIS, V ol. II. 127433.

1986. Plantas aromáticas da Amazonia, ler. Simposio do Trópico U mido, ANAIS, Vol. II 95-1 08.

1988, Contribuqáo ao conhecimento da Flora Medicinal de Roraima, SUPL. ACTA AMAZONICA, 18(1-2): 23-35.

-1988, Contribucáo a Flora medicinal de Mato Grosso do sul. SUPL. ACTA AMAZONICA, 18(1-1): 9-22.

DUKE, J.A., 1989. Foods as Pharmaceuticals, in: Simon, J.E., Kestner, A. and Buehrle, M.A., Eds. HERB'S 89, 166-176.

1990, Promising Phytomedicinals, in: Janick, J. Ed. EDVANCES IN NEW CROPS, Dioscorides Press, Portland, Dr. 
1991, Phytomedicinal, Forest harvest, (inedited).

1991, N eotropical nom-timber forest products, (inedited.).

LOPEZ GUILLEN, J.E. \& 1. KIYAN DE CORNELIO, 1974, Plantas medicinales del Perú, BIOTA IV, 10(77): 28-56.

PABON, E.M.A., 1982, Botánica económica de la Amazonía Colombiana, COLOMBIA AMAZONICA, Vol. 1,1:9-30.

VASQUEZ, M.R., 1989, Plantas útiles de la Amazonía Peruana 1195 P. (M imeografiado). 